\section{Getting from proto-sharing to sharing: collaborating on electronic solutions for ligand-binding assays}

\author{
"By collaborating we could avoid the wasteful use of resources, often \\ duplicating each other's efforts in developing electronic solutions to \\ support ligand-binding assay laboratory activities."
}

Keywords: bioanalytical $\approx$ collaboration $\approx$ electronic solutions $\approx$ ligand-binding assays " sharing m software

Have you ever noticed how toddlers share an object? They let others examine and even touch the object but actually never let go. This 'protosharing' [1] is unfortunately a lot like how bioanalytical ligand-binding assay (LBA) laboratories share their software, automated methods or electronic solutions that they have developed. We like to talk about them, even present them at conferences about what we have developed, but never quite let go. If we are going to collaborate on developing electronic solutions, we will need to learn to share.

With pharmaceutical and biotechnology companies under pressure from both shrinking budgets and the constant need to find areas to improve efficiency, it may be time to explore whether bioanalytical LBA laboratories should consider collaboration operating models for developing their electronic solutions for processes including laboratory automation and data management. Unlike laboratories with chromatographic data systems and LC-MS instrumentation, there is no standardization for automated-data workflows in LBA laboratories. While the current operating model is not broken, in the pursuit of efficiency and promoting innovation within the industry, this area should be re-examined. The current model is built on the premise that internally developed electronic solutions are proprietary and may even provide an advantage to one laboratory over another. But is this part of the deliverable of a bioanalytical LBA laboratory? Many would argue that the value of a LBA laboratory is not in its ability to develop electronic solutions, but to produce bioanalytical data and the information derived from the interpretation of the data. In this case, infrastructure, narrowly defined in this article as the resources to develop electronic solutions, without bioanalytical data lacks purpose. Collaboration could promote the adoption of electronic solutions in laboratories where development of electronic solutions is viewed as too resource intense and the development of more innovative solutions by sharing ideas and requirements for solutions. Not only would laboratory efficiency increase but interactions between sponsors and CROs could also benefit from an alignment of solutions. Alignment could lead to improved communication between CROs and sponsors, and potentially enhance a sponsor's confidence in a CRO's process owing to the familiarity with some of the electronic solutions. This collaboration model is one in which the entire LBA laboratory community could stand to benefit.

Companies have spent tremendous amounts of resources in establishing customized solutions, so it would not be surprising if many people would consider these solutions as proprietary. I would agree that highly customized solutions that are deeply integrated into a company's infrastructure are not in the scope of this discussion. In fact, collaborating on common electronic solutions would free up precious resources to focus on unique, company specific solutions. At the American Association of Pharmaceutical Scientists (AAPS) workshop on the 21st Century Bioanalytical Laboratory in Seattle, WA, USA, in 2009, where the focus was on the critical core functions of running an efficient bioanalytical LBA laboratory, there was a recognition that collaboration within the industry would be beneficial [2]. The topics covered included reagents, instrumentation, automation and electronic (software) solutions. Discussions with several workshop participants

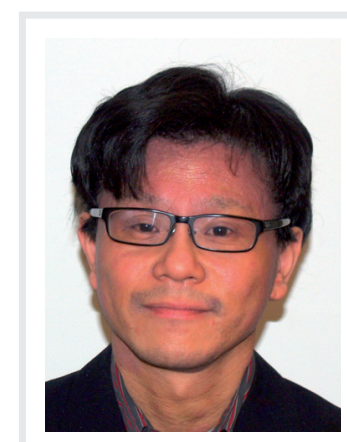

\section{Sheldon Leung}

Pfizer, Inc., I Burtt Road, Mailstop G200I, Andover, MA 02460, USA Tel.: + I 9782473282

Fax: + I 9782472842

E-mail: sheldon.leung@pfizer.com 
indicated a desire for a collaborative process for developing open-sourced electronic solutions. Adoption of this idea would require a very large shift in the current paradigm. Therefore, the aim of this editorial is to bring this topic to a larger audience and to encourage discussions around finding a way to collaborate around electronic solutions.

So why share? What would be the benefit? By collaborating we could avoid the wasteful use of resources, often duplicating each other's efforts in developing electronic solutions to support LBA laboratory activities. Even if a collaborative solution did not meet all of one's desired requirements, being able to leverage off the solution would be advantageous. Another positive outcome of sharing would be the ability to draw upon a larger pool of ideas, requirements and potentially previous experiences or solutions, to develop an improved product that benefits all laboratories.

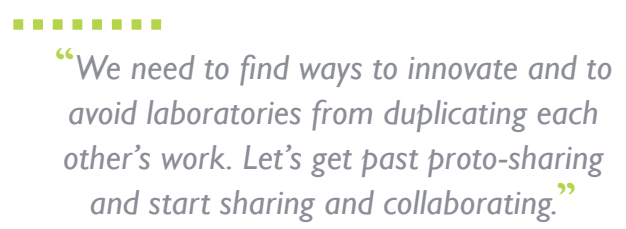

What could we collaborate on? Incorporating new technology often requires data translators in order to input the raw data into data analysis software or laboratory information management systems (LIMS). All companies need to develop translators if the latest technology is not compatible, which is a very common occurrence due to proprietary data formats. The eSolutions team formed at the AAPS workshop on the 21st century bioanalytical laboratory is working towards getting the LBA community (end-users and vendors) to adopt an automated data interchange process that could alleviate this issue. However, this is a long term solution, and in the meantime, this issue continues to be a hurdle for laboratories adopting new technologies. The programming of automated immunoassay methods is also repeated in multiple laboratories despite the fact that the actual liquid-handling procedures are more than likely almost identical. The effort required to adapt the developed method to one's specific set of instruments or even integrating new technology is relatively minor in comparison to developing the entire method. Collaborations could also be large-scale projects such as automating the entire workflow of a bioanalytical laboratory, including sample receipt, sample analysis and data reporting. Collaborating on large-scale projects would be where this effort would yield the maximum return on investment. This pooling of resources could help laboratories overcome the inertia of implementing significant changes to their workflow that could dramatically improve efficiency.

While there are plenty of examples of pharmaceutical consortiums, examples of collaboration outside of the research arena are hard to come by. This of course is not surprising; however, one might expect that coming to an agreement on sharing research data might be more complicated than sharing electronic solutions. However, precedent for infrastructure collaboration does exist outside of the pharmaceutical industry. An interesting collaboration to highlight has been recently established and is made up of nonprofit and public organizations whose aim is to lower government IT costs by sharing software that governments have developed. This consortium called 'Civic Commons' recognizes the wastefulness of governments developing similar software and see sharing software as a way to spread innovation throughout governments [3].

What would collaboration amongst LBA bioanalytical laboratories look like? Consider the Civic Commons model. Initially the consortium will look to create a database of available technology with the goal of relicensing the technology to allow for sharing. Civic Commons would then maintain the applications as well as a directory of the applications. Government entities could then search the database to identify solutions they need. New applications would be added to the database as they are developed and identified. Applying this to LBA laboratories, one proposal would be for participating companies to share their solutions, thereby creating a pool of solutions from which companies could draw from. However, it would be naive to think that this model would work for the pharmaceutical industry. Unfortunately, this model does not provide enough incentive for companies who have already invested heavily in electronic solutions, to share their developed solutions. In addition, this would require some form of management to ensure equality of what is deposited and withdrawn, as well as to maintain the solutions to ensure access. A simple solution might be to collaborate on establishing common requirements for new solutions, which 
could then be put out to bid for vendors to develop a solution. If one of the requirements is for the solution to be open source, then the solution will be open to all companies, thereby advancing the industry as a whole.

I have only just touched upon areas for collaboration and potential ways to collaborate. I believe there is plenty of substrate to discuss on this topic. In the effort to improve LBA laboratory efficiency, we need to find ways to innovate and to avoid laboratories from duplicating each other's work. Let's get past proto-sharing and start sharing and collaborating.

\section{Websites}

1 Baby center.

www.babycenter.com/404_how-can-i-teachmy-toddler-to-share_6823.bc

\section{Acknowledgements}

The author would like to thank Bonita Rup, Boris Gorovits and Chad Ray for their thoughtful discussions on this topic.

\section{Financial \& competing interests disclosure}

The author is an employee of Pfizer, Inc. and owns stock options in Pfizer, Inc. The author has no other relevant affiliations or financial involvement with any organization or entity with a financial interest in or financial conflict with the subject matter or materials discussed in the manuscript apart from those disclosed.

No writing assistance was utilized in the production of this manuscript.

2 AAPS Workshop: 21st Century Bioanalytical Laboratory.

www.aapspharmaceutica.com/meetings/

workshops/21stcentury/index.asp
Civic Commons. http://civiccommons.org 